Einführung in das Schwerpunktthema

\title{
Transitionsstrategien in Richtung Nachhaltigkeit
}

$\mathrm{M}$ Von Jan Nill ehr als 15 Jahre nach Beginn der Nachhaltigkeitsdebatte ist die Liste der ungelösten ökologischen Herausforderungen immer noch beträchtlich. Wie der folgende Beitrag von Vellinga und Wieczorek eindrücklich zeigt, kann bei überregionalen und vor allem globalen Umweltproblemen von einer Entkopplung von Umweltverbrauch und Wirtschaftswachstum keinesfalls die Rede sein, von einer Minderung des Umweltverbrauchs, wie sie etwa im Bereich Treibhausgasemissionen für nötig gehalten wird, ganz zu schweigen. Zudem scheint das Potenzial von einfachen ökologisch-ökonomischen Win-Win-Lösungen inzwischen weitgehend ausgereizt. Die zunehmende Skepsis gegenüber Selbstverpflichtungen oder die Härte der Auseinandersetzung um die Einführung des Emissionshandels sind eindeutige Indizien.

Ein ökologischer Strukturwandel steht folglich weiterhin oder wieder auf der Agenda. Gleichzeitig hat sich zunehmende Skepsis gegenüber dem Erfolg politischer Steuerung ,von oben“ breit gemacht. Die Grenzen des Ordnungsrechts sind hinlänglich bekannt und um die politische Durchsetzung der ökonomischen AlternativRichtschnur „Preise sollen die ökologische Wahrheit sagen" steht es kaum besser. Sie ist angesichts der gesellschaftlichen und wirtschaftlichen Widerstände selbst gegen die vergleichsweise harmlose real existierende ökosteuer sowie den Emissionshandel zur bloßen Floskel verkommen.

Doch nicht überall herrscht politische Tristesse und akademische Ideenflaute, wie ein Blick über den Tellerrand der deutschen Debatten zeigt. In den letzten Jahren wurden insbesondere auf europäischer Ebene eine Reihe von politischen Strategieansätzen entwickelt, die einen Ausweg aus dem skizzierten Nachhaltigkeitsdilemma zu weisen versuchen: Strategisches Nischenmanagement, Transition Management und Zeitstrategien lauten die inzwischen in der wissenschaftlichen Fachdebatte einschlägigen, in der weiteren Öffentlichkeit und deutschen Politik jedoch fast unbekannten Konzepte. Letzteres zu ändern sowie die Chancen und Grenzen der neuen Konzepte zu diskutieren, ist Ziel des vorliegenden Schwerpunkts.

\section{Die Beiträge im Überblick}

Zunächst stellt René Kemp das in den Niederlanden entwickelte Konzept des Transition Management vor. Im Zentrum stehen Systeminnovationen zur Erreichung möglichst konsensual festgelegter gesellschaftlicher Nachhaltigkeitsziele. Er geht auch auf erste Erfahrungen mit der politischen Umsetzung ein, denn Teil des laufenden vierten niederländischen Umweltpolitikplans sind Transitionsstrategien in den Problemfeldern Energie, Verkehr und Landwirtschaft. Klaus Jacob setzt sich aus politikwissenschaftlicher Perspektive wohlwollend kritisch mit dem Konzept auseinander und fragt nach seinen Erfolgsbedingungen. Manche seiner Anmerkungen beanspruchen auch für den neu entwickelten Ansatz der Zeitstrategien ökologischer Innovationspolitik Gültigkeit, der im Anschluss näher vorgestellt wird. Christian Sartorius und Stefan Zundel argumentieren für eine dynamische Perspektive: Politische Akteure sollten sich aus den Innovationsdynamiken ergebende Zeitfenster nutzen und gezielt auf deren Entstehung hinarbeiten. Was das in einzelnen Handlungsfeldern konkret heißen kann, wird von Daniel Weiner und Stefan Zundel am Beispiel Kunststoffrecycling, von Rüdiger Haum und Jan Nill am Beispiel energieeffiziente Wohngebäude und von Christian Sartorius am Beispiel mobile Brennstoffzelle illustriert. Eine erste Einschätzung der Erfolgschancen von Zeitstrategien aus Sicht der umweltpolitischen Praxis liefert der Beitrag von Peter Franz. Ein etwas älteres Konzept, das einen Baustein im Rahmen eines Transition Management darstellen kann und aus zeitstrategischer Sicht vor allem als Strategie zur Vorbereitung von Zeitfenstern anzusehen ist, ist das strategische Nischenmanagement. Bernhard Truffer stellt das im Rahmen eines EU-Projektes entwickelte Konzept vor und erläutert es an einem Beispiel aus dem Bereich Verkehr.

Gemeinsam ist allen präsentierten Ansätzen, dass sie die begrenzten Möglichkeiten einer Top-DownSteuerung in komplexen Gesellschaften und kapi- talistischen Wirtschaftssystemen akzeptieren. Stattdessen versuchen sie, am Kern wirtschaftlicher Veränderungsdynamiken, den Innovationen, anzusetzen und sich diese mit geeigneten Strategien politisch nutzbar zu machen. Dahinter verbirgt sich die Hoffnung, dass durch die Dynamik schöpferischer Zerstörung neue, längerfristige Win-WinMöglichkeiten geschaffen werden können, die das Erreichen von Nachhaltigkeitszielen erlauben. Dass eine solche Wirkung auch an politische und gesellschaftliche Veränderungen gebunden ist, wird im zeitstrategischen Ansatz versucht, durch die Beschäftigung auch mit politischen und sozialen Zeitfenstern zu berïcksichtigen. Wie wichtig dies ist, macht der abschließende Beitrag von Adrian Smith deutlich, der die Potenziale nischenorientierter Ansätze vor dem Hintergrund der historischen Erfahrungen mit den von der Alternativbewegung geschaffenen Nischen kritisch reflektiert.

Nichtsdestotrotz lässt sich aus den vorgestellten Ansätzen die Empfehlung ableiten, sich bei der Ausgestaltung von Nachhaltigkeitsstrategien nicht auf das Setzen ehrgeiziger Langfristziele zu beschränken, sondern auch die zur Erreichung solcher Ziele notwendigen Innovationsprozesse politisch zu gestalten (1). Wie realistisch das mit Transitionsstrategien in Richtung Nachhaltigkeit verbundene Governance-Modell im deutschen politischen System ist, kann sich dieses Jahr in mehrfacher Hinsicht erweisen. So stehen im Handlungsfeld Energie und Klimaschutz wichtige Weichenstellungen an. Zugleich bietet das diesjährige Zusammenfallen des von der Bundesregierung ausgerufenen ,Jahrs der Innovation“ mit der turnusgemäßen Evaluation und Weiterentwicklung der deutschen Nachhaltigkeitsstrategie ein politisches Zeitfenster für neue Ansätze.

\section{Anmerkung}

(1) Vgl. auch Nill, J./ Einacker, I./ Korbun, T./ Nordbeck, R./ Peine, A.: Nachhaltigkeitsstrategien. IÖW-Schriftenreihe 158/01, Berlin 2002.

\section{Der Autor}

Jan Nill ist wissenschaftlicher Mitarbeiter im Forschungsfeld Umweltökonomie und -politik am Institut für ökologische Wirtschaftsforschung (IÖW). Kontakt: IÖW, Potsdamer Str. 105, 10785 Berlin. Tel. 030-88459428, Fax 030-8825439,

E-Mail: Jan.Nill@ioew.de 
(c) 20I0 Authors; licensee IÖW and oekom verlag. This is an article distributed under the terms of the Creative Commons Attribution Non-Commercial No Derivates License (http://creativecommons.org/licenses/by-nc-nd/3.o/), which permits unrestricted use, distribution, and reproduction in any medium, provided the original work is properly cited. 